that training status influences the requisite dose and potential magnitude of strength development. Thus, it is important to establish the effect of resistance training versus combined resistance and aerobic training on muscular strength in specific populations using specific exercise doses. This may be so since those individuals seeking muscular strength development for basic strength or general health may need a much lower resistance-training dosage and lower training doses may limit or negate the interference effect. Unequivocal research makes it difficult to support the general concept of the interference effect as it seems to hold true in specific situations only and only when optimal strength development is required.

\title{
156 TARGETING BASIC STRENGTH: RESISTANCE TRAINING VERSUS COMBINED AEROBIC AND RESISTANCE TRAINING?
}

Brandon S Shaw Associate Prof \& Deputy Head of Department of Sport, Rehabilitation and Dental Sciences, Tshwane University of Technology, Johannesburg, South Africa

\subsection{6/bjsm.2010.078725.156}

Resistance and aerobic training are often performed concurrently in most exercise programmes in an attempt to get gains in more than one physiological system. This is done even though it is believed that combined resistance and aerobic training may impede the strength gains achieved by resistance training alone. However, this belief is not supported by unequivocal evidence and little is known about the effect of this mode of training on initial strength development or strength development for performance or health. It has been proposed that by simultaneously performing these two modes of exercise training, the strength gains achieved by resistance training alone may be impaired. Research has alluded to a possible optimal resistance training dose to optimise muscular strength development in certain populations and it seems 